\title{
Responses to crizotinib in a patient with c-ros oncogene 1 , receptor tyrosine kinase-positive advanced lung adenocarcinoma: A case report
}

\author{
NA ZHANG ${ }^{1,2}$, JIN-JI YANG ${ }^{1}$, XU-CHAO ZHANG $^{1}$, ZHI XIE $^{1}$, BIN-CHAO WANG $^{1}$, \\ HAI-YAN TU ${ }^{1}$, BEN-YUAN JIANG ${ }^{1}$ and YI-LONG WU ${ }^{1}$ \\ ${ }^{1}$ Guangdong Lung Cancer Institute, Guangdong General Hospital and Guangdong Academy of Medical Sciences, Guangzhou, \\ Guangdong 510080; ${ }^{2}$ Second Clinical Medial Committee, Southern Medical University, Guangzhou, Guangdong 510515,
} P.R. China

Received January 29, 2014; Accepted July 8, 2014

DOI: $10.3892 / 01.2014 .2571$

\begin{abstract}
Rearrangements to the c-ros oncogene 1, receptor tyrosine kinase (ROS1) gene are reported in 1-2\% of lung adenocarcinomas. These rearrangements are associated with a response to the small-molecule tyrosine kinase inhibitor crizotinib. ROS1 rearrangements can be detected using fluorescence in situ hybridization (FISH), which is considered the gold standard technique in detecting ROS1 rearrangements, and determining whether a patient would respond well to crizotinib treatment. However, FISH is an expensive and time-consuming assay, requiring specialized microscopy equipment and some level of technical expertise. The present report describes the case of a patient with advanced lung adenocarcinoma, who was identified to be negative for ROS-1 rearrangements by FISH, but positive by immunohistochemistry (IHC). The health of the patient improved following treatment with crizotinib. These results indicate that IHC assay could be an alternative option for the detection of ROSI gene rearrangements.
\end{abstract}

\section{Introduction}

C-ros oncogene 1, receptor tyrosine kinase (ROS1) gene rearrangements are reported in 1-2\% of lung adenocarcinomas and are sensitive to the small-molecule tyrosine kinase inhibitor crizotinib $(1,2)$. Fluorescent in situ hybridization (FISH) is the gold standard technique used for detecting ROS1-positive

Correspondence to: Professor Yi-Long Wu, Guangdong Lung Cancer Institute, Guangdong General Hospital and Guangdong Academy of Medical Sciences, 106 Zhongshan 2nd Road, Guangzhou, Guangdong 510080, P.R. China

E-mail: syylwu@live.cn

Key words: non-small cell lung cancer, gene rearrangements, receptor tyrosine kinase 1 , immunohistochemistry, crizotinib, partial responses adenocarcinomas and determining whether the cancer would respond well to crizotinib treatment. However, FISH is an expensive and time-consuming assay, requiring specialized microscopy equipment and technical expertise (1). Currently there are no reports of the use of immunohistochemistry (IHC) in the detection of ROS1 rearrangements in order to guide treatment of advanced non-small cell lung cancer (NSCLC). The present study reports the case of a patient with advanced lung adenocarcinoma who was ROS1-positive by IHC, but negative by FISH; the patient was determined to have had a partial response to crizotinib. Written informed consent was obtained from the patient's family.

\section{Case report}

A 36-year-old male presented to the Department of Oncology of Changzhou Tumor Hospital (Changzhou, China) with a persistent cough, which he had suffered with for two years, 1-day hemoptysis and shortness of breath. The patient was currently smoking 100 packets of cigarettes per year and had an otherwise unremarkable medical history.

A physical examination revealed decreased breath sounds in both lung fields, however no other signs were revealed. Positron emission tomography/computed tomography (CT) scans revealed a mass measuring $6.3 \times 6.2 \mathrm{~cm}$ in the right middle lung lobe, and the maximum standardized uptake value $\left(\mathrm{SUV}_{\max }\right)$ was measured as 9.4, with atelectasis of the right lower lobe. There were multiple ground-glass opacities (GGOs) in both lungs, the largest of which was positioned in the right apicoposterior segmental lung lobe, which measured $\sim 2.0 \times 2.5 \mathrm{~cm}$, and the $\mathrm{SUV}_{\max }$ was 2.4 . The supraclavicular lymph nodes were grossly enlarged on both sides. There was no metastasis observed in any other organ.

A fiber-optic bronchoscopy detected a stenotic lesion at the opening of the right middle lobe bronchus. A bronchoscopic biopsy confirmed the diagnosis of adenocarcinoma (Fig. 1A). Genotype analysis, using an amplification refractory mutations system, determined that the patient had expression of wild-type epidermal growth factor $(E G F R)$, a commonly mutated oncogene. FISH and IHC showed that the patient 

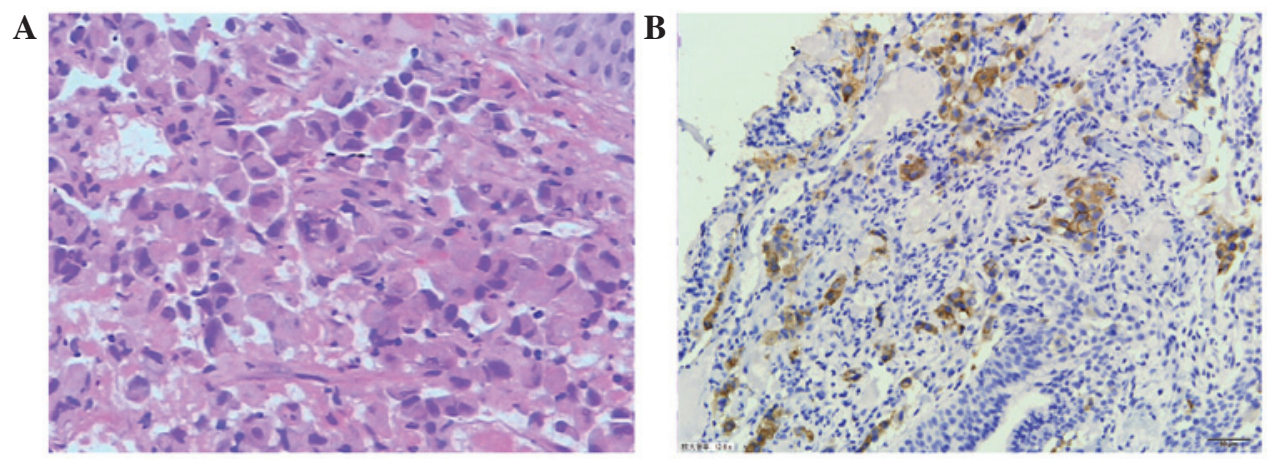

Figure 1. (A) Histological appearance of the bronchoscopic biopsy. Hematoxylin and eosin staining confirmed the diagnosis of adenocarcinoma. (B) Immunohistochemical analysis of c-ros oncogene tyrosine kinase receptor 1 protein expression. The staining intensity was measured as $>70 \% 2+$ or $3+$ (magnification, $\mathrm{x} 20$ ).

A

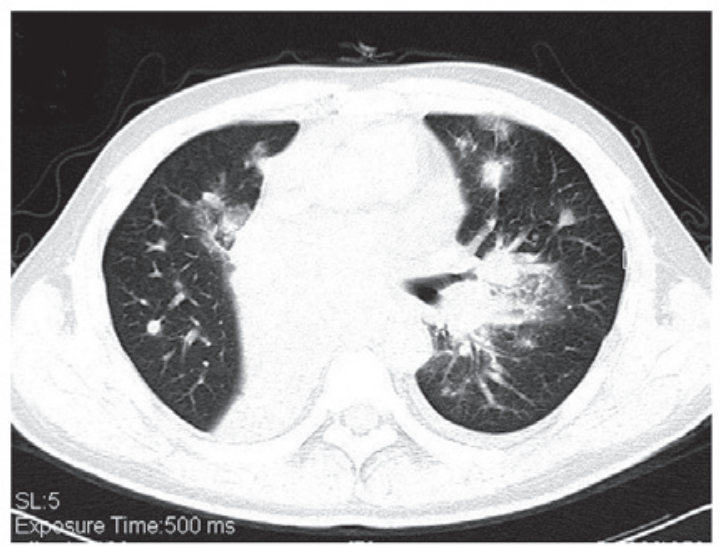

C

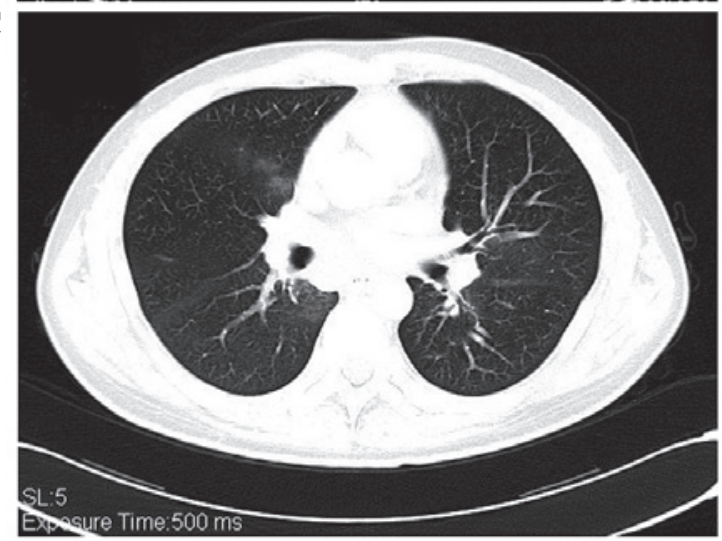

B

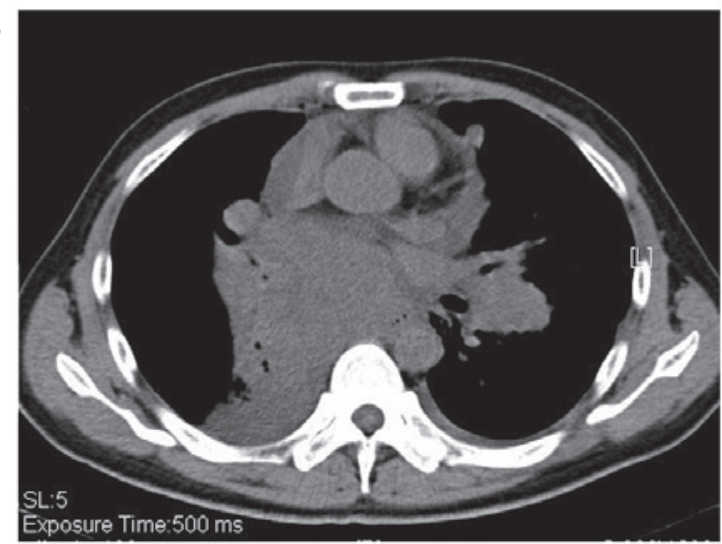

D

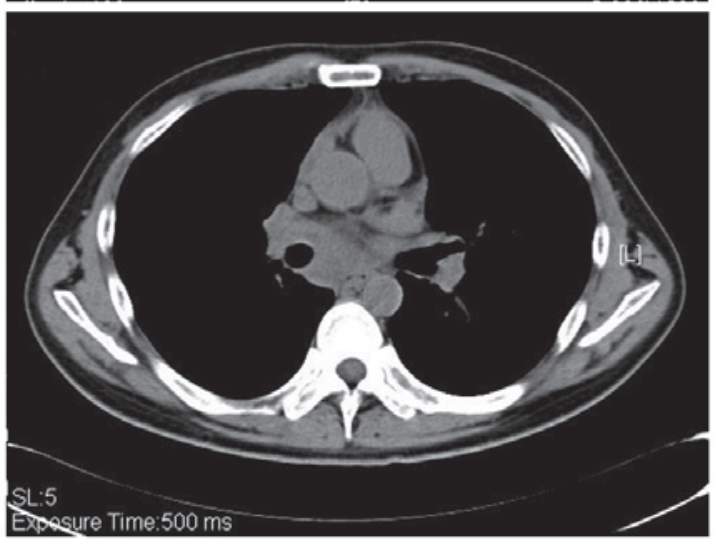

Figure 2. Computed tomography (CT) of the chest to observe the response to crizotinib treatment. CT scans of the chest were obtained (A and B) at baseline and (C and D) following 4 weeks of crizotinib treatment.
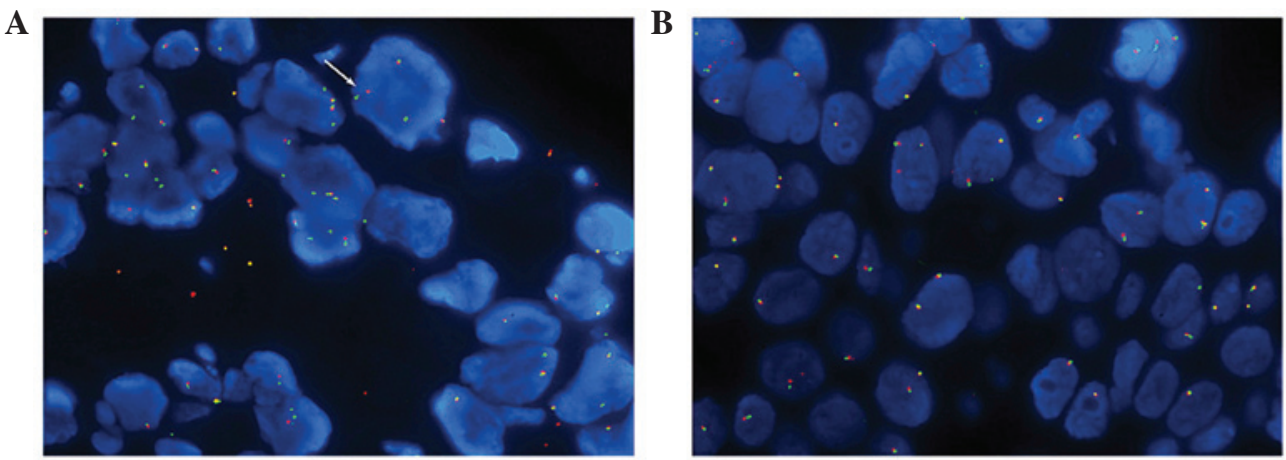

Figure 3. Fluorescence in situ hybridization analysis of c-ros oncogene tyrosine kinase receptor (ROS1) rearrangements. The adenocarcinoma was negative for ROS1 rearrangements at a cutoff value of 15\%. (A) Positive signal indicated (arrow; $>15 \%$ of the cells demonstrate split or single 3 ' signals). (B) Negative signal. 
was negative for the echinoderm microtubule-associated protein-like 4 (EML4)-anaplastic lymphoma kinase (ALK) fusion protein. The patient's clinical stage was determined as T4( Ipsi $_{\text {Nod }}$ ) N3M1a (contralateral lung) (stage IV).

One cycle of chemotherapy was administered, containing pemetrexed $\left(500 \mathrm{mg} / \mathrm{m}^{2}, \mathrm{~d} 1\right)$ and cisplatin $\left(75 \mathrm{mg} / \mathrm{m}^{2}, \mathrm{~d} 1\right)$. One month later, the patient's disease had progressed; the shortness of breath had worsened, and the pulmonary stenosis (PS) had deteriorated from 1 to 3 . The CT scans showed that the targeted lesion in the right middle lobe had shrunk,; however, the nodules in the left lung had become enlarged, and there was a new lesion measuring $\sim 1.0 \times 1.3 \mathrm{~cm}$ at the hilus of the left lung. The presence of GGOs in both lungs had increased and enlarged (Fig. 2A and B). The ROS1 protein was detected using IHC, with a staining intensity of $>70 \% 2+$ or $3+$ (Fig. 1B) (3). Furthermore, a break-apart FISH procedure was used to test for ROS1 gene rearrangements; however, the result was negative when the cutoff value was set at $15 \%$ (Fig. 3A and B) (4).

The patient had severe PS and was being treated with no other targeted therapies, therefore crizotinib treatment was orally administered at the standard dose of $250 \mathrm{mg}$ twice daily. One week later, the patient's symptoms had improved and the PS was measured at 1, a decrease as compared with the previous measurement. Following four weeks of crizotinib treatment, the CT scans detected an improvement; the targeted lesions in the right lung and left lung hilus had shrunk and the GGOs had almost disappeared (Fig. 2C and D). According to the Response Evaluation Criteria in Solid Tumors criteria, version 1.1 (5), the patient's cancer had elicited a partial response to the crizotinib treatment.

\section{Discussion}

Alongside mutations in the EGFR gene and EML4-ALK rearrangements, ROS1 rearrangements have been shown to define a new molecular subgroup in NSCLC (6). ROS1 shares a $49 \%$ amino acid sequence homology with EML4-ALK in the kinase domains, and several EML4-ALK inhibitors have demonstrated an inhibitory effect against ROS1 in vitro (7). Updated efficacy and safety data have been presented for the use of crizotinib in patients with advanced ROS1 rearrangements in NSCLC (8). An ongoing phase I clinical trial, investigating the effects of crizotinib (NCT00585195), enrolled patients with advanced ROS1-rearranged NSCLC, as determined by FISH. The patients receive a standard oral dose of crizotinib: $250 \mathrm{mg}$ twice daily. As observed in EML4-ALK-positive NSCLC, treatment with crizotinib has so far shown promising antitumor activity, with an overall response rate of $56 \%$ (2).
FISH is an expensive and difficult assay to support, requiring specialized microscopy equipment and technical expertise. By contrast, IHC is relatively cheap in comparison and can be performed in numerous pathology laboratories $(1,9)$. A previous study showed that if IHC thresholds of $2+$ and $3+$ are considered to indicate positive expression, ROS1 IHC is $100 \%$ sensitive and $92 \%$ specific for ROS1 rearrangements with FISH (1).

To the best of our knowledge, the present case report is the first to show positive ROS1-testing by IHC, but negative by FISH where the patient responded well to crizotinib treatment. These results provide evidence that IHC may be a useful screening method for the detection of ROS1 rearrangements, thus allowing for targeted precision therapy.

\section{Acknowledgements}

The present study was supported by the Key Lab System Project of Guangdong Science and Technology Department - Guangdong Key Lab of Lung Cancer Translational Medicine (China). This work was also supported by a grant awarded to Professor Yi-Long Wu, supplied by the National Natural Science Foundation of China (grant no. 81272618).

\section{References}

1. Sholl LM, Sun H, Butaney M, et al: ROS1 immunohistochemistry for detection of ROS1-rearranged lung adenocarcinomas. Am J Surg Pathol 37: 1441-1449, 2013.

2. Ou SI, Bang Y, Camidge DR, et al: Efficacy and safety of crizotinib in patients with advanced ROS1-rearranged non-small cell lung cancer (NSCLC). J Clin Oncol 31: 8032, 2013.

3. Kang W, Tong JH, Chan AW, et al: Yes-associated protein 1 exhibits oncogenic property in gastric cancer and its nuclear accumulation associates with poor prognosis. Clin Cancer Res 17: 2130-2139, 2011.

4. Davies KD, Le AT, Theodoro MF, et al: Identifying and targeting ROS1 gene fusions in non-small cell lung cancer. Clin Cancer Res 18: 4570-4579, 2012.

5. Eisenhauer EA, Therasse P, Bogaerts J, et al: New response evaluation criteria in solid tumours: revised RECIST guideline (version 1.1). Eur J Cancer 45:228-247, 2009.

6. McLeer Florin A, Mescam-Mancini L, Moro-Sibilot D, et al: Detection of ROS1 translocations in triple-negative lung adenocarcinomas. J Clin Oncol 31: 8099, 2013.

7. Ou SH, Tan J, Yen Y, and Soo RA: ROS1 as a 'druggable' receptor tyrosine kinase: lessons learned from inhibiting the ALK pathway. Expert Rev Anticancer Ther 12: 447-456, 2012.

8. Shaw AT, Camidge DR, Engelman JA, et al: Clinical activity of crizotinib in advanced non-small cell lung cancer (NSCLC) harboring ROS1 gene rearrangement. J Clin Oncol 30: 7508, 2012.

9. Cai W, Li X, Su C, et al: ROS1 fusions in Chinese patients with non-small-cell lung cancer. Ann Oncol 24: 1822-1827, 2013. 